\title{
Muy señor nuestro Alessandro Varaldo \\ La ricognizione nel mondo spagnolo e portoghese per riscoprire un autore italiano di successo ma dimenticato
}

\author{
Alessandro FERRARO \\ Università degli Studi di Genova ${ }^{1}$ \\ alessandro_ferraro@alice.it
}

Recibido: $15 / 06 / 2012$

Revisado: $21 / 01 / 2013$

Aceptado: 30/06/2013

\section{RIASSUNTO}

Questa è una prima ricognizione di ciò che è stato detto, adattato e tradotto di Alessandro Varaldo (1876-1953) nella Penisola iberica e in Sud America, che si sospetta siano gli unici territori stranieri dove si riesca a ritracciare qualcosa dell'autore italiano, ed è un'occasione per ripercorrerne la traiettoria letteraria che ha superato due transenne temporali come il 1900 e il 1950 ma non quella della dimenticanza.

Parole chiave: Varaldo, grottesco, diritto d'autore, Marinetti, «giallo».

Muy señor nuestro Alessandro Varaldo.

The Recognition in the Spanish and Portuguese World in Order to Rediscover a Famous but Forgotten Italian Author

\begin{abstract}
Who is Alessandro Varaldo (1876-1953)? This first analysis about what was said, adapted and translated of Varaldo's work in the Iberian Peninsula and South America, probably the only areas outside Italy where some traces of the Italian author can be found. It is a chance to go along a trajectory that has overcome two temporal barriers (1900 and 1950), even if it has not overtaken the oblivion's threshold.
\end{abstract}

Keywords: Varaldo, Grotesque, Copyright, Marinetti, Detective Story.

SOMMARIO: 1. Introduzione 2. Ardillas y lirones e il quasi grottesco 3. La definizione per legge del diritto d'autore 4. Marinetti e compagnia scrivente: il Gruppo dei Dieci 5. Juan Chabás e qualche considerazione 6. La massima onorificenza italiana e il maggior successo all'estero 7. La «probabilità» ed Estampas napoleónicas 8. Confidenze e conclusioni

${ }^{1}$ Universitá degli Studi di Genova - Dipartimento di Italianistica, Romanistica, Antichistica, Arti e Spettacolo (DIRAAS), Via Balbi 6, I-16126, Genova (Ge). 


\section{INTRODUZIONE}

Sui cartelloni dei 21 teatri di Madrid attivi nella stagione 1919-1920 apparvero 700 titoli di cui 216 al debutto, per un totale di 12.168 rappresentazioni, il $30 \%$ in più rispetto alla stagione precedente ${ }^{2}$. La maggior parte furono opere di autori spagnoli contemporanei, mentre ai classici del Siglo de Oro come Calderón de la Barca, Lope de Vega e Augustín Moreto rimase pochissimo spazio, poco agli stranieri sebbene non mancassero gli irrinunciabili Shakespeare, Molière e Goldoni, $\mathrm{o}$ i più recenti Wilde, Tagore e Ibsen.

Si parva licet componere magnis anche un altro straniero apparve in cartellone quella stagione: Alessandro Varaldo, autore di cui si cercherà di ripercorrere l'interessante, se non impressionante per quanto infaticabile e multiforme, percorso letterario che riuscì ad abbattere due barriere temporali più che indicative come il 1900 e il 1950. Già nella prima metà degli anni Quaranta però s'intravidero, nonostante le pubblicazioni postreme e postume, le prime ombre dell'oblio che lo avvolse in uno sproporzionato silenzio dopo un comunque sintomatico successo. Si parte qui dal suo debutto al Coliseo Imperial di Madrid perché l'occasione per presentare Varaldo attraverso i Cuadernos de Filología Italiana nasce con naturalezza dalla ricostruzione che si è tentata del riscontro che ebbe l'autore italiano nella Penisola iberica e in Sud America.

Quasi nessuno, oggi, saprebbe rispondere alla domanda "Chi è Alessandro Varaldo?". Eppure fu poeta simbolista nell'inseguimento di Gian Pietro Lucini e romanziere d'appendice con l'insegnamento di Anton Giulio Barrili (fra 800 e 900), nonché il primo a tradurre in italiano il Paradoxe sur le comédien di Diderot (1909) e critico drammatico per il Corriere di Genova (1903-1910), poi commediografo innovatore perché precursore del grottesco e di successo per tutti gli anni Dieci; fu il Direttore Generale della Società Italiana degli Autori (19201928) che, fra l'altro, definì per legge il diritto d'autore, e un membro del Gruppo dei Dieci che firmò l'epistolario enciclopedico Approcci (1928) e il romanzo collettivo Lo zar non è morto (1929) con la guida di Marinetti e la benedizione di Mussolini; fu uno scrittore di grande popolarità negli anni Venti con le sue storie d'amore e d'altri tempi e poi il padre fondatore del «giallo» all'italiana (Il sette bello, 1931), ma scrisse anche opere erotiche o per ragazzi, saggi e memorie.

\section{ARDILLAS Y LIRONES E IL QUASI GROTTESCO}

A Madrid nel 1920 il pubblico spagnolo conobbe il copione della commedia L'altalena di Alessandro Varaldo nel pomeriggio del 3 febbraio quando fu letto per la prima volta nella traduzione di Enrique Tedeschi e l'adattamento di José López Pinillos, con il titolo Ardillas y lirones, che felicemente sostituì quello italiano. Las ardillas sono

\footnotetext{
${ }^{2}$ Secondo il prezioso e particolareggiato studio di Dougherty e Viches (1990).
} 
gli scoiattoli mentre los lirones i ghiri, in mezzo la $y$ che congiungendoli li contrappone: da una parte le donne dall'altra gli uomini, e nello specifico le prime, ventenni ancora di belle speranze, che cercano di sposarsi per cominciare a vivere una vita più mondana $\mathrm{e}$ stimolante, mentre i secondi, trentenni sufficientemente di mondo, accettano di sistemarsi per sfuggire quella stessa vita che più che stimolante trovano già sfiancante. «Une femme se marie pour entrer dans le monde, un homme pour en sortir» recita la citazione di Hippolyte Taine in apertura del copione.

Enrique Tedeschi era certamente uno dei più convinti estimatori del teatro italiano d'inizio Novecento, del resto prima de L'altalena aveva già tradotto opere di Sabatino Lopez, Roberto Bracco e Dario Niccodemi. È innegabile però che fosse la firma di José López Pinillos a innalzare il valore dell'operazione, ad attirare l'attenzione: nel 1920 Parmeno (pseudonimo di Pinillos) aveva già pubblicato con successo tutta la sua prosa e il suo teatro, aveva già dato ampiamente prova della vivacità della propria penna come giornalista e del proprio pensiero come socialista.

L'attesa era quindi alta. Il Coliseo Imperial non risparmiò nelle spese, lo fece invece nei tempi: le prove cominciarono già il giorno dopo la lettura pubblica del copione, e l'intenzione era di rappresentare la commedia il prima possibile. Sfogliando le cronache teatrali di quei giorni si può ripercorrere, e si perdoni l'esagerazione, "il mese madrileno del teatro varaldiano": il 7 febbraio $E l$ Imparcial, il giornale in questo caso più puntuale, informò che le prove procedevano nel migliore dei modi, il 14 che c'erano problemi con la scenografia di José Martínez Garí, la settimana successiva che il debutto del 18 era rimandato ulteriormente per una protesta dei teatri. Infine mercoledì 25 febbraio 1920 un Coliseo Imperial tirato a lucido ospitò con gran lujo la prima di Ardillas y lirones, fra gli attori Manuel Vigo, Matilde Muñoz Sampedro e Maria Luisa Moneró. Applausi e repliche fino al 5 marzo.

Quasi tutti los diarios madrileni raccontarono dell'estreno della commedia: Época (1849-1936), La Correspondencia de España (1860-1925), El País (1887-1921), Heraldo de Madrid (1890-1939), El Imparcial (1896-1933), ABC (1903), Acción (1916-1924), El Sol (1917-1939) e La Libertad (1919-1939).

Generalmente i caratteri a piombo fissarono sulle pagine datate 26 febbraio 1920 il rispetto per il «signor» Varaldo, il «muy señor nuestro» come ne scrive cortesemente e formalmente Manuel Machado su La Libertad, e l'apprezzamento per l'originalità e l'ingegno, per il garbo. Inoltre la gratitudine verso Tedeschi per aver portato ancora una volta nel teatro spagnolo il meglio di quello italiano, o perlomeno, sempre per dirla con Machado, il «más viable y plausible» (Machado 1920), l'ammirazione per il lavoro di scrittura di Pinillos e la meraviglia per la messa in scena, dagli sforzi della produzione alle interpretazioni degli attori.

Di aria nuova e buon gusto si parlò sia ne El País che ne El sol. Sulla prima testata Arturo Mori definì Ardillas y lirones un «modelo de orginalidad literaria, de gracia, de refinamiento» e affermò che se fosse stato lecito ogni tanto elogiare la leggerezza quello era il caso perché la commedia aveva «todas las características de las obras modernas innovadoras y un superficialismo digno de todos los encomios» (Mori 1920). Con un lungo trafiletto José Alsina ne El sol confermò che 
Ardillas y lirones ruppe la routine dei cartelloni spagnoli, e aggiunse che la leggerezza della commedia se da una parte scoraggiava un coinvolgimento emotivo dall'altra assecondava un ingegnoso gioco letterario.

Un'opera atipica e appuntita che se a tratti impedì la comprensione non intiepidì la recezione. Riprendendo le parole di Manuel Machado, del resto il più importante fra $\mathrm{i}$ recensori della commedia, il pubblico «después de todo se mostrò contentísimo» (Machado 1920). Di succèse d'estime, probabilmente ancor più correttamente, raccontò Melchor Fernández Almagro, trasferitosi da appena due anni a Madrid ma non certo uno sconosciuto nel mondo della stampa spagnola, sulla prima pagina di Época ricordando inoltre che per quanto il pubblico spagnolo, soprattutto quello delle prime teatrali, amasse ritrovare nelle messe in scena il realismo più granitico o la caricatura più grossolana (dalla «brocha gorda») e si smarrisse davanti al nuovo, alla fine, a volte, sapeva apprezzarlo, premiando l'ingegno.

Il protagonista de L'altalena è Ugo, il domestico filosofo, il discendente del servus callidus, che ama la pace e che per la pace è disposto a fare e disfare qualsiasi cosa. Sia come critico drammatico sulle pagine del Corriere di Genova che come traduttore di Diderot nella prefazione al Paradosso sull'attor comico (Diderot 1909), oltre che in tutta una serie di articoli che arriva fino agli anni Quaranta, Varaldo aveva già dimostrato la sua preferenza e la profonda conoscenza per il ruolo di brillante e quindi non poté non concentrare tutte le proprie forze espressive nella creazione del domestico che legge Schopenhauer, commenta Leopardi e traduce Platone. Un personaggio per qualche tempo indimenticato grazie anche a chi gli prestò il volto: in Italia Alberto Giovannini e Ruggero Ruggeri, mentre in Spagna Carlos, il sostituto spagnolo di Ugo, fu interpretato da Manuel Vigo, anche in quest'occasione «excelente actor» come scrisse Manuel Machado.

Il giorno dopo aver visto in azione Carlos, Alsina chiamò in causa Figaro, personaggio caro a Varaldo che avrebbe, non a caso, poi scritto un articolo dal titolo «Caduta e resurrezione di Figaro» su Comoedia nel 1931 (Varaldo 1931b), nel quale, pur tentando di minimizzarne l'aspetto rivoluzionario ne evidenzierà l'importanza, convinto della necessità, storica e scenica, di ogni tempo e società, che chi sta nel gradino più basso, semplificando, si prenda gioco (che è prendersi una rivincita) di chi sta su quello più alto. Nel prologo di una commedia successiva a L'altalena, Il medico delle anime, Varaldo attraverso un personaggio spiega come un burattinaio nella storia del mondo non sia mai mancato, e come non sia il più potente ma il più riflessivo. Lo stesso Ugo è consapevole della sua superiorità.

Per la felicità di un padrone occorre che il servo gli sia superiore d'ingegno e di sapere. Quando esistevano i veri padroni, cioè quando esisteva la schiavitù, non era cosa rara acquistare per servo un essere superiore per nascita, senno e raffinatezza, che fosse nutrito di filosofia [...]. Lo schiavo, attento e previdente, studiato il carattere del padrone, adulava o eloquentemente taceva, secondo che lo credeva opportuno [...]. Il servo insomma conosceva il padrone, mentre il padrone non si dava la pena di conoscere il servo, dal che derivava che il servo era il vero padrone, $\mathrm{e}$ il padrone assai meno d'un servo. (Varaldo 1939: 269-270) 
Sin da subito è chiara la scelta di Varaldo di far parlare al servo una lingua immodesta e insolita che lo caratterizza e innalza rispetto agli altri, tanto da incuriosire chi lo sente forbito filosofo mentre lo vede umile domestico. Incuriosire o indispettire come nel caso di Giulio, il vecchio nobile di casa, padre di Giacomo e Norina, che soffrendo uno scomodo senso d'inferiorità nei confronti di Ugo lo tratta con scherno e sgarbo, quasi fosse ogni volta un attacco preventivo in difesa di un'aristocrazia antiquata quanto annacquata.

E se è vero com'è vero, però, che addirittura Gramsci (1953: 336) prese ad esempio, e bersaglio, Varaldo per condannare un certo tipo di teatro disimpegnato, si può comprendere che, né con L'altalena né con nessuna ulteriore prova (Gramsci si riferisce nello specifico ad Appassionatamente del 1917) ${ }^{3}$, fu primo interesse del commediografo insistere sull'aspetto sociologico e potenzialmente sovversivo delle sue storie. Quella di Varaldo è una posizione essenzialmente conservatrice nella vita e puramente intrattenitrice nell'arte, il suo interesse è quello di creare ogni volta un personaggio irresistibile e porlo al centro di un intreccio efficace, e soprattutto sperimentare quel bizzarro borghese fatto di certe caricature e forzature che rende L'altalena un testo precursore del genere grottesco. Sempre nella sfera del disimpegno, e forse proprio per questo, Varaldo prende qualche distanza da parte della tradizione teatrale italiana riproponendone deformati alcuni pilastri, come il triangolo adulterino, o personaggi e ambienti con parodie per corroderne $\mathrm{i}$ principi, con ironia per sgretolarne l'ipocrisia.

El interés de esta comedia, más que en el pensamiento que dista mucho de ser nuevo, está en su desarollo y sobre todo en la figura del criado filósofo, que es quien dirige los acontecimientos y que da á la obra cierto sabor de humanismo recordando a los esclavos y a los parásitos mentores y consejeros de sus amos y patrones, que hallamos en la comedia clásica. 「... \ Esta figura señala el tono irónico y fino de la comedia, abocetada en algunas escenas, exagerada en otras hasta rayar en la caricatura, pero con todo ingeniosa y nueva, en relación con los tipos de comedia que monopolizan neutro escenarios. (Fernandéz Almagro 1920)

Giacomo è un giovane agiato e annoiato, insoddisfatto e inquieto, che pensa sia arrivato il momento di abbandonare gli eccessi e scegliere una moglie, che sarà Giovanna, amica della sorella di Giacomo, Norina, la quale intanto complica la vicenda non poco quando comincia a prender lezioni da Ugo anche se un'istitutrice l'ha già, cioè Ester, e a invaghirsi del servo colto che un'amante l'ha già, la stessa Ester, così Norina sfinita dalla gelosia scoppia e rivela tutto a suo padre Giulio. Mentre Giacomo non riesce a gestire, come se non bastasse e com'é prevedibile, il matrimonio con Giovanna che entra in crisi e, dopo le ultime rivelazioni, il rapporto con Ugo, il quale, dopo le ultime rivelazioni, farà le valigie per un ostracismo vintage o un esilio volontario, lasciando la casa, esaurendo la commedia e

3 La recensione della commedia Appassionatamente di Alessandro Varaldo apparse sull'Avanti! del 22 novembre 1918. 
richiamando il sipario. Sipario che calerà però solo dopo un'ulteriore discussione fra Giovanna, ventenne novella moglie di Giacomo, e Massimo, trentenne futuro marito di Norina:

E perché mostrarsi stanco della vita passata, che è quella in cui la fanciulla entra e che crede propria in avvenire?

[...] Norina si sposa per entrare nel mondo, voi per uscirne. Vi mentite in buona fede, ambedue vantando la vostra vita abituale, quello che conoscete e di cui vi innamorate a vicenda, voi della quiete, della pace, della monotonia, lei dell'avventura, delle festa continue, dei piaceri mondani. E così giuocate all'altalena. Oggi la menzogna, domani l'infelicità. (Varaldo 1939: 283-284)

Il grottesco è il terzo punto di forza della commedia, dopo il domestico filosofo e la vicenda ingarbugliata, e con questi s'intreccia e irrobustisce. «Las ardillas van a emparejar con los lirones y tal unión va a resultar monstruosa» (Alsina 1920), di questa Ugo è artefice e vittima.

Ne L'altalena datata 1910 si abbozzò un grottesco ancora embrionale ma che anticipò di qualche anno quello di Enrico Cavacchioli de La campana di vetro e soprattutto di Luigi Chiarelli de La maschera e il volto. Non con l'ufficialità con cui è considerato padre del «giallo» italiano, ma di essere il primo fra i pionieri del teatro grottesco a Varaldo glielo hanno riconosciuto tutti, i suoi contemporanei e gli studiosi successivi. Fra questi Gigi Livio, Paolo Puppa, e Luigi Squarzina per il quale Ugo è un "“meneur de jeu" che non può non ricordare vagamente [il] Capocomico nei Sei personaggi in cerca d'autore» (Squarzina 1987: 96-97).

Non stupirà dunque che la fortuna di Varaldo drammaturgo sia legata principalmente a L'altalena, commedia «arditamente paradossale, originale nella concezione come nella struttura» (Simoni 1931), per usare le parole di Renato Simoni, un critico teatrale notevole e contemporaneo di Alessandro Varaldo come Marco Praga, che scrisse invece di Un marito innamorato: «Quando udrete la commedia vedrete che Alessandro Varaldo, con un'arte sottile e sapiente, sa farvi accettar tutto per buono» (Praga 1921: 251).

Se all'inizio del 1920 a Madrid debuttò l'adattamento de L'altalena, a dicembre dello stesso anno a Torino ci fu la prima proprio di Un marito innamorato, e qualche mese dopo Leonardo Marini ne parlò positivamente sullo spagnolo Cosmópolis, riprendendo in qualche modo l'opinione di Praga.

Il marito innamorato è Marco, la moglie amata è Claudia che scatena tutte quelle insicurezze del protagonista alle quali s'aggroviglia la commedia. Se è vero che Claudia è molto più giovane, che si è sposata per amore e non per interesse, che è sempre stata fedele, allora Marco pensa di indurla al tradimento per perdonarla e renderla debitrice, per cercare con un disperato gesto di sfilarle il coltello di mano per impugnarlo lui dal manico, disposto a sacrificare il corpo della moglie per averne l'anima, insomma, come dice Marini «ingenuo, por no decir idiota» (Marini 1921: 320). Poi il pentimento e il bacio, un lieto fine che non scongiura la sciagura latente perché «a menos que llegara Mefistófeles a rejuvenecer a Fausto, la tragedia estará siempre preparada para surgir» (Marini 1921: 321). 
Nello stesso numero di Cosmópolis fu citata la raccolta di novelle Moralità immorali come opera che aggiunse nulla e nulla tolse a un autore che continuava a farsi leggersi con piacere. Si concluda però l'analisi de L'altalena, ripercorrendone le tappe cronologiche significative, in Italia, in Spagna e in Argentina. Per Varaldo il passaggio dalle colonne del Corriere di Genova, col quale collaborava come critico drammatico e letterario dal 1903, alle assi del palcoscenico fu accompagnato dal giornale stesso che nei giorni precedenti ricordò a più riprese l'appuntamento del 22 luglio 1910 al Politeama Regina Margherita, e profetizzò un teatro delle grandi occasioni. Varaldo era già un motivo di richiamo e un certificato di garanzia, specialmente a Genova, città che lo seguiva sui giornali e lo apprezzava per i suoi romanzi, che l'aveva conosciuto come seguace simbolista di Lucini per accettarlo poi come erede naturale di Barrili: «egli sembrava il beniamino della parte colta e intellettuale della città» (Parodi 1953: 36).

In effetti fu un evento, e non effimero: un pubblico numeroso assistette alla prima rappresentazione de L'altalena, che sarebbe poi uscita in diverse edizioni su rivista $\mathrm{e}$ in volume, che sarebbe stata riproposta in diverse città d'Italia e dalle migliori compagnie ancora per un trentennio abbondante, per riformularsi felicemente nelle trasmissioni radiofoniche degli anni Quaranta. Parallelamente a Madrid, un anno dopo il debutto a teatro, nel marzo del $1921 \mathrm{fu}$ pubblicata Ardillas y lirones nei popolari fascicoli della rivista Los contemporáneos con la copertina illustrata da Yzquierdo Durán, e nel maggio del 1922 la commedia fu citata ancora nei necrologi di Pinillos come uno degli esempi della sua poliedrica carriera. Inoltre nel settembre del 1928 debuttò nei teatri oltreoceano nella versione argentina $\mathrm{Mi}$ criado y yo della compagnia di Enrique De Rosas, Eva Franco e Matilde Rivera.

\section{LA DEFINIZIONE PER LEGGE DEL DIRITTO D'AUTORE}

Dopo Ardillas y lirones, Varaldo apparse sulla stampa spagnola grazie al coinvolgimento nel Gruppo dei Dieci, di cui si parlerà più avanti, e prima ancora, ma in maniera più circoscritta, per l'importante ruolo istituzionale di Direttore Generale della Società Italiana degli Autori che ricoprì dal 1920 al 1928, mentre il Presidente era Niccodemi e Presidente Onorario D'Annunzio.

Si apra a proposito, anche se pretestuosa, una piccola parentesi. S'immagini la quotidianità di un dirigente che «entra nel suo studio vastissimo, sgombro e netto, come una lizza pronta pel torneo [...]. Tutta la corrispondenza, piena di beghe, di cavilli, di questioni, di richieste, di proteste, di minacce» (De Angelis 1925: 729). Lo definì «un lavoro spaventoso» in una lettera a Silvio d'Amico datata già 29 gennaio $1921^{4}$, gravoso aggravato da «mezzo personale nuovo e mezzo malato», privo di spirito d'iniziativa e di capacità non ordinarie, come specificò a Nino

${ }^{4}$ Dalla lettera di Alessandro Varaldo a Silvio d'Amico del 29 gennaio 1921 conservata nell'archivio della Biblioteca Museo dell'Attore di Genova (MA). 
Martoglio, in un'altra lettera di sfogo di qualche giorno dopo ${ }^{5}$. «Il Grand'ufficiale Varaldo governa tutti, [...] trova rimedi, corre ai ripari, risponde a tutte le sollecitatorie, acconsente a raccomandare, a presentare, ad aiutare tutti. Poi le visite, ininterrotte, d'ogni specie di uomini e di donne. Intanto il telefono e gli ordini urgenti agli impiegati» (De Angelis 1925: 729).

Per avere delucidazioni, per rivendicare tutele o chiedere aiuti, per amicizia rinnovata o semplice reverenza, gli scrisse, come si apprende dalla lettura di moltissime missive, quasi tutto il mondo delle lettere e dei teatri di quegli anni: Amelia Rosselli, Ettore Petrolini, Alfredo Testoni, Salvator Gotta, Guido da Verona, poi Lopez, Benelli, Govi, Zacconi e Ruggeri (benché alcuni di questi, gli ultimi citati per esempio, siano corrispondenti anche del Varaldo non uomo di potere).

Oltre all'ultimo saluto a Roma al corteo funebre verso Asolo di Eleonora Duse (1923), alla trasferta parigina con Pirandello per la prima francese dei Sei personaggi (ancora nel 1923), o alla settimana mondana per attendere La figlia di Iorio di D'Annunzio al Vittoriale (1927), Varaldo s'occupò di faccende più impegnative come la promozione dei giovani autori attraverso l'Ente Teatro Sperimentale che coinvolse il Comunale di Bologna e il Manzoni di Milano.

Si segnali, perché si cerca di essere il più inclusivi possibile, un aneddoto raccontato nel 1922 su El Imparcial da Enrique Tedeschi, colui che due anni prima aveva tradotto in spagnolo L'altalena: si trovava sull'espresso Milano-Roma quando nella stazione di Corbezzi, in Toscana, che non era una sosta prevista, il capostazione prima ordinò al macchinista di frenare, nello stupore generale, e poi corse con un pacchetto sotto braccio cercando Varaldo fino a trovarlo nel suo scompartimento e consegnargli ciò che gli premeva consegnargli.

Varaldo, ya desenvuelto el paquete sobre sus rodillas, pudo satisfacer la curiosidad exasperada de sus compañeros de tren. [...] El incomparable jefe de Corbezzi, enterado de que aquella tarde pasaba por delante de su estación, se le había ocurrido detener el expreso para entragarle un drama en prosa y verso y en cinco actos, que durante los ocios de su cargo había ido sacándose de la cabeza. (Tedeschi 1922)

Non furono nulla gli impegni elencati fin qui paragonati a ciò che a metà mandato Varaldo dovette gestire, perché la pazienza per leggere un dramma del capostazione di Corbezzi è poca cosa in confronto alla responsabilità di definire per legge il diritto d'autore. Mentre furono tutti respinti, per resistenze governative o per esigenze corporative, i progetti presentati nei mesi precedenti, il memorandum consegnato nel maggio del 1925 da Varaldo a Mussolini ebbe ben altro destino. Il "Progetto Varaldo", come lo chiamò la stampa, è così sintetizzabile: il cosiddetto creatore è afflitto dalla lacunosità delle leggi e dall'indifferenza degli esattori; si cerca, pertanto, sia di porre rimedio difendendo qualsiasi forma d'arte, sia di semplificare e automatizzare ogni formalità amministrativa per la protezione dell'opera d'arte, protetta per cinquant'anni (oggi per settanta) non dalla sua data di

\footnotetext{
${ }^{5}$ Dalla lettera di Alessandro Varaldo a Nino Martoglio del 3 febbraio 1921 (MA).
} 
pubblicazione, ma dall'anno di morte dell'autore; inoltre si consente il riacquisto dei diritti sulle opere prive di tutela per mancanza del loro deposito presso il competente Ufficio del Registro, da quel momento obbligatorio. Soprattutto fu ribadito il concetto generale che pubblico significasse profitto.

In un articolo apparso su La Libertad dall'eloquente titolo "; Sociedad de autores o de recaudadores?», Luis Alcázar senza mezzi termini definì esattori i dirigenti della Società giacché - «el comer y el rascar todos es empezar» (Alcázar 1924) - non smettevano di accumulare profitti su profitti, e invitò i sindacati italiani, nel caso specifico dei cinematografi, a essere meno intransigenti perché Varaldo e compagnia dirigente sembravano "insaziabili". Era una poltrona scomoda quella del Direttore Generale e non fece bene all'immagine di Varaldo (quasi dipinto come lo sceriffo di Nottingham), ma a posteriori non si può non riconoscergli capacità di mediazione e concretezza d'azione per una definizione per legge del diritto d'autore ancora valida dopo quasi novant'anni.

Per quanto però si sia rivelato vincente, fra l'aprile del 1925 (consegna del memorandum a Mussolini) e il settembre del 1926 (entrata in vigore della legge sul diritto d'autore), il percorso del "Progetto Varaldo" fu più che inquieto: non solo perché D'Annunzio, dopo aver accettato di riscriverlo elevandolo a grande carta civile, a punto di riferimento europeo e a quant'altro le ambizioni del Duce e del Poeta insieme potessero puntare, lo restituì con poche note a margine e con due mesi di ritardo, ma piuttosto per lo scoppio di una «piccola guerra (civile) della Società degli Autori» (Lucignani 1984) che vide contrapposta una serie d'importanti intellettuali guidati da Giuseppe Brunati a quella che lui definiva l'alleanza affaristica «trevesiana mondadoresca varaldesca giordanesca».

Si chiuda questa parentesi così come si è aperta: con un aneddoto spiritoso nel suo corrispettivo in spagnolo. Questo è quello che fu riportato dall'argentino Caras y Caretas qualche mese dopo essere apparso in italiano nell'Almanacco Mondadori del 1927, quando ormai si poteva scherzare sulla questione:

Lucio D'Ambra, Alejandro de Stefani, Corrado Alvaro, Rosso de San Secondo, Orio Vergani y Pirandello hacían antesala en la secreteria de la Sociedad de Autores Italianos.

Alejandro Varaldo dice: de autor.

$-i$ Sabes quiénes son esos seis? Pues... seis personajes en busca de los derechos

Nel 1928 Varaldo lasciò, con un'eredità quindi non banale, il posto di Direttore Generale a un gerarca fascista. L'abbandono fu quello di un uomo addolorato e, dai pochi documenti realmente privati di cui si dispone, si comprende come alla sua costante inclinazione a sentirsi vittima di complotti si sommò una più concreta insofferenza per l'ormai insostenibile ingerenza. Sentiva ostacolata la propria efficienza, fra direttive ricevute in carta bollata e favori richiesti a mezza bocca, minata la propria autonomia all'interno di quella Società Italiana degli Autori che era ormai divenuta eloquentemente Ente Italiano per il Diritto d'Autore. 


\section{MARINETTI E COMPAGNIA SCRIVENTE: IL GRUPPO DEI DIECI}

Non fu Varaldo però un antifascista. Leggendo le lettere inviate a Mondadori (il carteggio maggiormente consistente e costante dell'autore) ${ }^{6}$ non si incontrano raramente accenni a Mussolini, dai complimenti al direttore del giornale La Libertà Camillo Aymard perché autore del libro d'esaltazione del Duce alle rassicurazioni sul progetto Storie e leggende napoleoniche, volume d'implicita propaganda.

Dopo la prima parte della seconda guerra mondiale, invece, solo sospiri di sollievo, sia nelle missive sia sui giornali, dall'articolo omaggio all'attore antifascista Roberto Bracco dell'agosto 1943 in cui Varaldo allude a un periodo opprimente e gioisce per una libertà ritrovata, marcando già il sommario confine fra l'errore e la sua cancellazione, fino al più rilassato articolo Tragico e buffo figuro datato ormai 1947 in cui assicura come Mussolini non avesse «mai una opinione propria: l'ultimo che parlasse aveva ragione, e spesso a distanza di ore diede istruzioni che si smontavano a vicenda», come fosse un tragicomico capopopolo colui che prima proibiva Ben-Hur, approvato dalla censura, per poi pentirsene, o più semplicemente scordarsene, («Ma come vi è venuto in mente di vietare quel film?»), il tragicomico capopopolo che «s'innamorava delle parole, e le lanciava come catapulte, senza essere sicuro del significato» come quella volta che per insultare un ministro esclamò «é un morfologico» (Varaldo 1947a). Intanto però lo stesso Varaldo nel 1928 aveva firmato insieme ad altri nove scrittori questo telegramma indirizzato a Mussolini:

Dieci romanzieri italiani et fascisti uniti da questa sera in Gruppo d'azione per servire il Romanzo italiano in Italia ed all'estero devotamente salutano il Duce meraviglioso augurando che dai romanzi dell'Era Fascista esca un giorno il poeta della nuova epopea come già dalla storia sparsa del martirio in camicia rossa uscì nella Marcia trionfale il creatore di una più grande Italia ${ }^{7}$.

È questo il primo documento relativo al Gruppo dei Dieci, il primo di pochi. Non si fosse avuta la possibilità d'avere fra le mani Approcci, il primo (e unico mai uscito) tomo dell'epistolario enciclopedico dell'amore, Il Novissimo segretario galante, del 1928, quasi introvabile, o il romanzo precocemente collettivo, decisamente riuscito, Lo zar non è morto, del 1929, comunque ripubblicati di recente (Gruppo dei Dieci 2005, 2011a), si stenterebbe a credere all'effettiva esistenza del Gruppo per quanto abbia contorni surreali. È possibile però far luce sul progetto e sul suo prodotto: con la combattività di Marinetti e la benedizione di Mussolini quello dei Dieci fu un fenomeno fascista e letterario, con un gesto serioso Milano.

${ }^{6}$ Il carteggio è conservato presso la Fondazione Arnoldo e Alberto Mondadori di

7 Telegramma del Gruppo dei Dieci a Benito Mussolini, datato 24 maggio 1928 e conservato presso l'Archivio Centrale dello Stato (Fondo S.P.D.C.O. 509.446). 
e discutibile crearono comunque un epistolario spassoso e un ottimo romanzo. Queste alcune delle parole di Marinetti nella prefazione a Lo zar non è morto:

La grande Italia fascista deve non soltanto realizzarsi politicamente, militarmente, industrialmente, commercialmente e colonialmente, come sta facendo sotto l'occhio vigile del Duce, ma deve anche esprimersi. Perché l'Italia abbia la sua alta luminosa espressione nel mondo occorre mettere in primo piano la letteratura e specialmente il romanzo che, col teatro, può sviluppare la maggiore potenza italianizzatrice. (Marinetti 1929: 7)

In ordine alfabetico Antonio Beltramelli, Massimo Bontempelli, Lucio D’Ambra, Alessandro De Stefani, Filippo Tommaso Marinetti, Fausto Maria Martini, Guido Milanesi, Alessandro Varaldo, Cesare Viola e Luciano Zuccoli, questi gli scrittori così diversi fra loro, lontani «miliardi di chilometri» ma uniti da «alcuni scopi di patriottismo artistico» (Marinetti 1929: 7), che nel luglio del 1928 sedettero intorno a un tavolo insieme a diplomatici e aristocratici e ad alcuni altri, come dire, nunzi di nazioni estere, per l'atto costitutivo del Gruppo dei Dieci.

Fra gli invitati quello che qui interessa è senz'altro Rafael Sánchez Mazas: in Italia negli anni Venti come corrispondente, sposò un'italiana per poi tornare in Spagna e divenire consigliere di José Antonio Primo de Rivera, fondatore, nel giro di un lustro, della Falange Española, di cui Sánchez Mazas fu ideologo e promotore.

In un articolo apparso su $A B C$ il 24 luglio 1928 in cui, per la verità, si dilungò un po' troppo nella descrizione storica ed estetica della location, Sánchez Mazas raccontò dell'evento che si svolse ovviamente a Roma capitale, a Villa Madama, grazie alla disponibilità dei conti Dentici di Frasso, di un Marinetti aizzatore «con su palabra joven, caliente, animadora, más simpática cuanto más oída» (Sánchez Mazas 1928b: 7), della firma di un atto ufficiale dopo un elegante buffet e della proposta ruffiana di chiamare Edizioni di Villa Madama quelle che poi furono più semplicemente le Edizioni dei Dieci (nei volumi si legge Sapientia con sede a Roma, reale nello spazio di un paio di frontespizi, un po' meno nei fatti).

«Para España, las conclusiones son halagüeñas en extremo» (Sánchez Mazas 1928b: 7) scrisse Sánchez Mazas soddisfatto dell'attenzione dimostrata dagli italiani giacché quella spagnola, insieme all'inglese, fu scelta con voto unanime come lingua principale per la diffusione delle opere dei Dieci all'estero. In generale però si fece tutta una serie di promesse (poi non mantenute): promozione di giovani talenti nazionali, aiuti finanziari agli editori interessati, premi letterari e scambi internazionali. Si fantasticava anche che i Dieci potessero presto divenir quindici, venti, tutti pronti a rivoluzionare il mondo letterario, e far trionfare il fascio littorio. La questione di un ricco premio per un giovane talento ebbe qualche eco all'estero ancora per un anno abbondante grazie alla pubblicazione del programma del Gruppo in appendice a Lo zar non è morto, e in Spagna ne parlarono, fra gli altri, Gaceta Literaria e l'Heraldo de Madrid.

Pubblicato nel 1929 ma in progetto già nel 1927, come si evince da un altro articolo redazionale dell'Heraldo de Madrid uscito il 26 settembre, quindi quasi un 
anno prima del simposio romano di Villa Madama (a confermare la travagliata e ancora misteriosa gestazione del Gruppo), Lo zar non è morto è il groviglio d'intrighi internazionali e incredibili inseguimenti, da Pechino a Parigi, passando per le stanze segrete del Vaticano, di coup de théâtre e di scene gangster, con una fascinosa femme fatale fascista per non farsi mancare nulla.

«Questa eterogenea collaborazione, una volta tanto, ad un romanzo di avventure non vuole dare nessuna direttiva artistica» (Marinetti 1929: 7). L'unione qui ha davvero fatto la forza. I Dieci hanno scritto un capitolo a testa, mentre l'altra cinquantina insieme: alcune forzature fascistizzanti o le frequenti discrepanze toponomastiche sono pecche di poco conto davanti al ritmo e all'ironia; si tratta d'ingegneria infallibile e incredibile a pensare quante mani abbiano lavorato al romanzo precocemente collettivo (Luther Blisset e Babette Factory sarebbero arrivati settant'anni più tardi). Nato per l'intrattenimento è riuscito nel proprio intento.

De Lo zar non è morto non rimane quasi nulla oggi, se non il desiderio di saperne di più o più semplicemente ed emozionalmente di ritrovare una delle venticinque copie autografate da tutti i Dieci, premio per chi si fosse classificato fra la quinta e la trentesima posizione al concorso allegato in cui s'invitavano i lettori ad associare ognuno degli autori al proprio capitolo. Ancora più misterioso l'altro progetto del Gruppo: Il Novissimo segretario galante, una sorta di epistolario enciclopedico, 400 lettere d'amore «per tutte le evenienze». Furono pensati sei volumi che avrebbero dovuto ricalcare l'evoluzione di una relazione sentimentale e accompagnare, aiutandoli, gli innamorati senza idee, com'è evidente dai titoli: Approcci, Primo bacio, Gelosia, Guai dell'amore, Lontananze e ricordi e Rotture.

Introdotto da una prefazione incalzante e iperbolica, delirante e demolitrice di matrice marinettiana e firmata da tutti i Dieci (che cosa sono la Divina commedia, Delitto e castigo e I promessi sposi in confronto, si chiedono), Approcci è composto da 99 lettere in cui, parafrasando qualche titolo, si danno suggerimenti a una «ammiratrice romantica» per avvicinarsi a un «uomo illustre» come a un «giovinetto inesperto» per «una signora amica di casa, della mamma e dei parenti», fino a più specifiche e spassose occasioni: Lettera a signora veduta al Palatino in compagnia di un signore anziano che sembrava guardare languidamente lo sconosciuto che la seguiva e che ora le scrive o Lettera la mattina dopo un ballo, nel quale si è bevuto molto e del quale si ha un ricordo incerto, a signora che è stata compagna alla festa, per limitarsi a qualche esempio.

Lo stesso Rafal Sánchez Mazas sempre su $A B C$ dedicò tre colonne al volume appena uscito di cui passò in rassegna molte lettere, un utile manuale redatto dai dieci italiani «generosos y geniales» nel quale «las coyunturas más pintorescas, imprevistas y delicada han sido tenidas en cuenta» (Sánchez Mazas 1928a: 7).

Nonostante quest'oggetto pronto all'uso cerchi di piegare anche l'approccio sentimentale alle leggi del progresso, cerchi di semplificarne i meccanismi e diminuirne le tempistiche, si proponga di esser per l'amore ciò che il telegrafo fu per le comunicazioni, risulta un epistolario che riconsegna un mondo lontano, un diverso modo d'intendere, confronto a quello odierno, il rapporto fra i due sessi e il rispetto 
per la donna. Regala anche una godibile gamma di esercizi di stile; alcuni casi limite sono virtuosismi di vocabolario, fantasia e buone maniere; la fruibilità del manuale è garantita dalle risposte che seguono le rispettive missive: così, se Approcci consiglia cosa scrivere suggerisce anche come replicare.

Sebbene le lettere siano firmate con nomi fittizi o semplici «devotissimo», nell'indice ne è segnalato l'autore. Varaldo scrive cinque scambi, la prima è Lettera per accompagnare un mazzo di fiori in occasione di una festa, a una signora con la quale si ha poca dimestichezza (Gruppo dei Dieci 1928 [2011a]: 20-22), ed è subito una prova d'intelletto e galateo del mittente nella speranza che il linguaggio dei fiori non tradisca qualche doppio senso, lui che si accontenterebbe di un sorriso e una stretta di mano. Il signore della seconda lettera di Varaldo (Gruppo dei Dieci 1928 [2011a]: 144-147), invece, vorrebbe qualcosa di più dall'amata collega d'ufficio dopo un corteggiamento anonimo ma serrato, le ha messo le violette nel bicchiere, temperato il lapis e ripulito la gomma e, addirittura, tutti i giorni le ha pulito il tavolo non fidandosi dell'usciere: come non meritare una passeggiata dopo un'estenuante giornata d'ufficio? Peccato che il corteggiatore sia imbranato e la risposta un inappellabile rifiuto:

Signore, lei mi ha rivelato un arcano. Tutti i mesi do cinque lire all'usciere perché mi metta dei fiori nel mio bicchiere. Siccome i fiori sono suoi, ciò vuol dire che l'usciere mi ha rubato le cinque lire. Ho preso una fiera ramanzina dal mio capo ufficio per una cancellatura sulla mia copia, ciò vuol dire che la gomma non era abbastanza pulita. Ho voluto prendere un appunto sopra una lettera e i due lapis si sono spuntati, ciò vuol dire che non erano ben temperati. La prego dunque di non farmi spendere invano cinque lire con l'usciere, di non farmi prendere sgridate dal capo ufficio e di lasciarmi l'orgoglio di sapere temperare i miei lapis meglio di lei.

Naturalmente io vado a casa uscendo dall'ufficio, ma non vado mai sola, perché viene a prendermi il mio fidanzato. Lei non se ne è mai accorto, perché probabilmente mi aspetta all'angolo sinistro della porta del'ufficio, mentre il mio fidanzato naturalmente mi aspetta all'angolo destro. L'unica cosa in cui andiamo d'accordo, è nel non prendere il tram.

Con ciò le ricambio i più distinti saluti (Gruppo dei Dieci 1928 [2011a]: 147).

C'è decisamente più feeling fra i due protagonisti di un accettato invito a un tè in casa del mittente interessato (Gruppo dei Dieci 1928 [2011a]: 154-157): la destinataria, nonostante la timidezza, si è lasciata convincere perché le donne misurano il garbo di un uomo dalla quantità di bugie che riesce a dire. Un'altra avance fallita è quella della quarta lettera ideata da Varaldo (Gruppo dei Dieci 1928 [2011a]: 220-224), e se la signora non andrà al parco col signore probabilmente non sarà solo perché, la sera prima, lui ha definito «indigesta» una commedia che a lei è invece piaciuta. Si concluda il resoconto dell'apporto di Varaldo ad Approcci, singolare nel panorama dell'autore ma esemplare del volume in questione, con una lettera ambigua (Gruppo dei Dieci 1928 [2011a]: 327-331): un signore molto influente scrive a una signorina senza appoggi molte righe con un po' di sana 
spocchia per riceverne una singola di assenso. L'amore non sempre può brillare di luce propria.

Ecco una delle tante citazioni che precedono le lettere di Approcci, questa scelta da Varaldo: «In amore non c'è di bello che l'inizio. Non stupisco che si ricominci sì spesso e con tanto piacere». Due opere, quelle dei Dieci, che, con strumenti diversi, sono accumunate da ciò che potrebbe esser definita la cifra del gruppo: l'ironia. Non prendendo troppo sul serio il grande romanzo d'avventura e la tradizione epistolare, Marinetti, Varaldo e compagnia scrivente hanno affidato al tempo, che è stato però meno galante di loro, due volumi godibili anche oggi.

\section{JUAN CHABÁS E QUALCHE CONSIDERAZIONE}

Che l'Italia di Mussolini esercitasse comunque un fascino culturale sulla Spagna di Miguel Primo de Rivera è ormai fuori dubbio e due esperti di letteratura spagnola del Novecento, Luis Rodríguez Puértolas e Javier Peréz Bazo, lo confermano e lo spiegano. Il primo definendo l'attenzione della critica come conseguenza della «toma de poder por el fascismo italiano en 1922 y la instauración de la dictatura primorriverista en 1923 「quel despertaron en España el interés por el nuevo fenómeno que comenzaba a producirse en Europa» (Rodríguez Puértolas 1986: 64), Peréz Bazo partendo proprio dalle parole di Rodríguez Puértolas, individua come immediato precedente «la resonancia que tuviera la traducción en 1919 del manifesto marinettiano nada menos que con el crédito de Gómez de la Serna» (Peréz Bazo 1992: 180), ça va sans dire più rilevante della risonanza che ebbe un decennio dopo il pur marinettiano "gesto dei Dieci".

Molti critici finirono per esser cronisti banali o commentatori servili, e Rafael Sánchez Mazas può essere un esempio di quelli che «terminarían rindiendo pleitesía a sus dogmas con servidumbre de acólito», come scrive Peréz Bazo, che fa il nome di Juan Chabás il cui caso «ha de comprenderse netamente distinto y, por supuesto, debe excluirse de las algaradas patrioteras o elogios de quienes fueron propensos a la atracción del fascismo» (Peréz Bazo 1992: 180).

Juan Chabás, scrittore e soprattutto studioso appassionato della letteratura italiana contemporanea, è probabilmente chi con più frequenza e attenzione si occupò di Varaldo in Spagna, ed è utile partire dalle sue parole per ragionare sulla produzione romanzesca dell'autore italiano. Se si sfogliano le pagine del saggio Italia fascista del 1928 - col quale colmò un vuoto con l'«único libro español útil sobre el fascismo italiano en sus aspectos políticos y culturales» come affermò La Gaceta Literaria segnalandolo immediatamente ${ }^{8}$ - si può notare come Chabás intenda gestire la materia chiamando in causa, oltre a Svevo e Pirandello, tutta una serie di scrittori cosiddetti secondari, preferendo Bontempelli, Jahier, Slataper, Panzini e Puccini, rispetto a Gotta, D'Ambra, Saponaro, Zuccoli e Varaldo

\footnotetext{
${ }^{8}$ Juan Chabás, «Italia fascista», La Gaceta Literaria, 37 (1 de julio 1928: 3).
} 
«únicamente representativos de una copiosa producción novelesca para lectores burgueses: para una clase media, algo letrada y aficionada a que le cuenten historia, ya de sabor galante, ya casi blancas, todas de un gran sentimentalismo postromántico» (Chabás 1928: 206).

Queste ultime parole di Chabás non si possono però smentire, e ciò che scrisse di Varaldo nello specifico non è da sottovalutare. Il primo accenno che s'incontra è su La Libertad in un omaggio a Ceccardo Roccataglia Ceccardi, «hombre nacido para no ser sino poeta» che visse e amò la «ciudad tal vez menos literaria de toda Italia». Perché per lo spagnolo di Alicante non era letteratura a Genova il dannunzianesimo di Mario Maria Martini né «las crónicas locales» (Chabás 1926) di Varaldo, riferendosi nel 1926 probabilmente a Il fior d'agave edito un anno prima. Sembra un po' incoerente che appena cinque mesi dopo, recensendo il romanzo Il covo sempre di Varaldo (1927a), lo stesso Chabás, ancora su La Libertad, affermasse che le due opere migliori dell'autore fossero La costa azzurra (Varaldo 1920c) e quel Genova sentimentale (Varaldo 1913b) - che uscito nel 1913 costituisce, con le sue memorie cittadine, la prima delle tre parti proprio de Il fior d'agave - chiosando che Varaldo fosse per la Liguria ciò che Ferdinando Paolieri era per la Toscana.

Chabás comunque coglie ne $\mathrm{Il}$ covo la tensione sottile ma effettiva dell'autore al rinnovamento. Con questo romanzo Varaldo - che «a los cincuenta años posee una labor abundantísima, de muy desigual valor, pero siempre hábil y jamás desnuda de una cualidad que si no es la esencial de un novelista, es, desde luego, de las más importantes: una fácil, fluida amenidad» (Chabás 1927b: 6) - sentendo il bisogno di battere nuove strade, dalla Liguria si spostò in Toscana. Il romanzo attraversato da «toda la emoción de una inquietud sincera» è pregevole proprio lì dove è manchevole. Varaldo sembra in difficoltà non potendo utilizzare il linguaggio ligure, non potendo contare sugli appigli della propria terra, sul suo portolano di riferimento, e alla fine «no se libra de la epidemia del sueño y del recuerdo», come scrive, con l'aiuto di Freud, Chabás che così conclude la recensione: «Pero Il covo sigue siendo una novela distraida e interesante, a pesar de esas indecisiones constructivas de su autor, picado por el ansia de renovarse. Hay, sobre todo, en sus páginas un misterio de sensualidad aventurera y febril, que las [sic] otorga mucha italianidad. En este punto Varaldo ha conseguido su propósito» (Chabás 1927b: 7).

Com'è successo con la messa in scena di Ardillas y lirones per il miglior teatro, con un aneddoto e una polemica per la direzione della Società Italiana degli Autori, con il racconto di un evento e qualche altro accenno per l'esperienza del Gruppo dei Dieci, si prenda ora la recensione de $I l$ covo come pretesto. Pretesto in questo caso per parlare della prosa di Varaldo in generale, dei pregi e dei difetti aggiornati alla fine degli Trenta, dopo una bibliografia già lunghissima, di cui i titoli migliori sono La bella e la bestia (Varaldo 1919a), i tre volumi de I re magi - L'amante di ieri (Varaldo 1925a), La grande passione (Varlado 1920a) e L'ultimo peccato (Varaldo 1920b) - e i tre de La marea - Il falco (Varaldo 1922), I cuori solitari (Varaldo 1921) e Mio zio il diavolo (Varlado 1913a). Questo è ciò che scrive il critico milanese Enrico Piceni a proposito de Il covo su La bancarella delle novità: 
Come ognuno sa, i dieci comandamenti... sono undici. L'undicesimo l'ha dettato or non è molto Alessandro Varaldo ad uso e consumo degli scrittori: non annoiare il pubblico tuo. E siamo sinceri: esempio più unico che raro fra i legiferatori, l'autore di Mio zio il diavolo è il primo ad obbedire alla sua legge.

[...] Narratore scaltro, egli non ambisce ad esplorare abissali profondità psicologiche, né a raggiungere translucidi effetti di stile. Unico suo scopo, l'ha più volte lui stesso apertamente dichiarato, è quello di raccontar bene storie interessanti: e lo raggiunge sempre.

Anche l'ultimo romanzo Il Covo è uno di quei libri che se vi capitano tra le mani in una sera disoccupata e solitaria, possibilmente in campagna, e quando già potete contare sopra un buon bicchiere di vino e una sigaretta morbida [...] vi risolve lì per lì il problema di passar piacevolmente qualche ora. Che se poi, fuori è freddo, e al libro, al bicchiere, alla sigaretta, sia aggiungerà anche un caminetto cordiale con un po' di bragia da tormentare e fissare a lungo fantasticando fra capitolo e capitolo, la vostra felicità sarà perfetta.

[...] Quanto a me Il Covo me lo sono letto in treno; e, non fosse stata la sollecitudine di un cortese compagno di viaggio, non mi sarei certo fermato al paese dov'ero diretto. (Piceni 1928: 52-54)

Pubblicato da Mondadori nel 1927, il romanzo racconta di un ragazzo che non può rifiutare di fare il precettore presso una nobile famiglia toscana, lui che non è pratico, che è povero e vagabondo per incoscienza e sfortuna, anche se la proposta viene da un individuo poco raccomandabile e il castello dove dovrebbe lavorare è poco rassicurante, scenario poi di un amore sincero ma sventurato. In sintonia con Enrico Piceni un altro critico milanese, Eligio Possenti, che aggiunge, sempre a proposito de Il covo, che «Varaldo ama che i suoi libri appartengano a quelli che si leggono d'un fiato e ne avvia le vicende al modo delle girandole» (Possenti 1927).

Il covo è un classico nell'universo varaldiano: attorniato da una sempre ricca schiera di personaggi tipizzati, il protagonista, preferibilmente un giovane con esperienze di vita che lo legittimino moralmente, agisce in città spesso settentrionali ben conosciute e descritte o in castelli possibilmente isolati e misteriosi, somiglianti alle famiglie che li posseggono, con dannazioni attorcigliate intorno al proprio albero genealogico, poi indizi e premonizioni sparsi per lo svolgimento della trama ma anche nell'arredamento degli interni, concessioni al fantastico, amori inconfessabili quando non impossibili, contrasti marcati e spesso manichei, duelli e delitti, ma più facilmente semplici intrighi, giochi delle parti e rapidi scambi di battute, ambientazioni naturali in primo piano, e condizioni meteorologiche che partecipano dei sentimenti dei protagonisti, i quali più raramente si mostrano attraverso analisi psicologiche, una superficialità che è scorrevolezza, una corsa che s'interrompe in un equilibrio finale che può escludere il lieto fine.

Perfetta macchina dell'intrattenimento di pagine da leggere tutte d'un fiato, Il covo è l'esempio di come, per usare ancora le parole di Possenti, Varaldo ormai conosca «le ingenuità del pubblico, la freschezza delle sue impressioni più facili, i sentimenti per i quali è di pronta vibrazione, gli episodi che lo soggiogano. Si potrebbe dire che è un libro scritto con scaltrezza, se non lo fosse con sincerità» (Possenti 1927). 
Certo Varaldo è morso dal desiderio di rinnovarsi («picado por el ansia de renovarse», si è già citato Chabás) ma la sua ricerca non nasce quasi mai da esigenze espressive impellenti quanto dal rispetto dei propri propositi, come l'allenamento della fantasia (la «decima musa») e la lotta contro la noia (l'«undicesimo comandamento») così, senza travagli, «i suoi libri respirano un'aria di vacanza e di ristoro; in un certo senso contengono una sottile gioia di esser nati. Hanno, per prima cosa, divertito l'autore. Sono venuti su a poco a poco, foglio su foglio, stalattiti di carta, con la pazienza di uno stillicidio» (Possenti 1927).

Tornando più strettamente a Juan Chabás si prenda la sua segnalazione su $L a$ Gaceta Literaria dell'Almanacco Mondadori 1927 nella quale definisce quella dell'almanacco un'operazione utile per l'editore oltre che dilettevole per il lettore e in particolare riflette sul resoconto di un concorso per cercare talenti lirici: di 234 manoscritti furono apprezzati solo quelli di tre giovani poeti, che se uno aveva un nome altisonante, Apollinaro Duce, l'altro decisamente e dantescamente non scherzava, Filippo Argenti, mentre il terzo, Vittorio Gerace, venne premiato dopo qualche dubbio fosse meglio non premiare nessuno. Per Chabás un disastro, e il discorso era delicato, un problema italiano essendo ormai difficile trovare «un poeta nuevo, con interés y alta conciencia de lo que debe ser la poesía después de los futuristas, los crepuscolares, pascolianos y d'annunzianos» (Chabás 1927a). Chabás guardava alla Mondadori, tutto sommato apprezzata per gli sforzi, però Vallecchi nel 1919 aveva pubblicato Allegria di naufraghi e Gobetti nel 1925 Ossi di seppia.

Il punto che qui interessa è che Varaldo per Chabás, con Saponaro e Gotta ancora una volta, ma con l'aggiunta addirittura di Marino Moretti, era la testimonianza vivente, e cinquantenne, che Mondadori non aveva giovani in catalogo, l'esempio di quegli autori di cui certo importava ancora ciò che dicevano e facevano, per parafrasare la rubrica dell'Almanacco intitolata Quel che dice e quel che $f a . .$. , ma che non potevano più far fronte alle esigenze di un pubblico moderno. In conclusione, Chabás sperava in un rinnovamento più deciso dell'editrice di Milano confidando in Umberto Fracchia e Giuseppe Antonio Borgese.

\section{LA MASSIMA ONORIFICENZA ITALIANA E IL MAGGIOR SUCCESSO ALL'ESTERO}

Nel 1931 Alessandro Varaldo dimostrò, però, di saper ancora far fronte al cosiddetto pubblico moderno, e con il genere più moderno di tutti: il «giallo». Si continui questa prima ricognizione del riscontro rapsodico che ebbe Varaldo nel mondo iberico e sudamericano, che ormai si è compreso essere la segnalazione delle tappe italiane più interessanti del suo percorso, arrivando alla produzione poliziesca che caratterizzò tutti gli anni Trenta di Varaldo in Italia e altrove quando, per esempio, la Livraria do Globo pubblicò i primi e migliori «gialli» Mondadori partendo da Il sette bello del 1931 che in portoghese divenne $O$ sete belo nel 1935, pretesto per parlare di Alessandro Varaldo attraverso la sua onorificenza massima: 
padre fondatore del «giallo» italiano. Poi, anche con ulteriori editori stranieri, fu la volta di altri polizieschi Mondadori: Le scarpette rosse (Varaldo 1931c), La gatta persiana (Varaldo 1933b) e La scomparsa di Rigel (Varaldo 1933c).

A qualsiasi ragionamento sulla questione sia permesso anteporre qualche utile, e forse piacevole, accenno al rapporto d'amicizia fra Alessandro Varaldo e Arnoldo Mondadori. Queste le parole della figlia dell'editore dei «gialli»:

Molto più divertente per noi ragazzi Alessandro Varaldo, il famoso giallista, che veniva ospite tutti gli anni per otto giorni a Meina con la moglie bionda. Venivano a settembre, nella settimana che chiamavamo la "settimana dei Varaldo", perché appena arrivavano cominciava a piovere e smetteva quando partivano.

In queste tetre, terribili giornate di pioggia, Varaldo organizzava per noi recite e film gialli. [...] Quello che ricordo meglio, perché mi procurò una grande angoscia (tanto per cambiare), aveva come personaggio di centro la mamma, nel ruolo di una vedova abbindolata da un losco figuro, che era Alberto [...]. La legge era rappresentata da Giorgio e Tullio Cimadori, nei panni di due ragazzi che avendo intuito l'andazzo criminoso chiamavano di rinforzo il detective, cioè Varaldo. Ecco Varaldo arrivare a tutta velocità sull'Alfa dello zio Bruno a spiegare il mistero, demenziale, imbrogliatissimo, con la storia di un bambolotto sparito e di me che avevo rubato la marmellata.

L'anno dopo vollero fare le cose in grande. Alberto pretese la sceneggiatura, il ciak ecc. [...] Un altro anno abbiamo fatto Tarzan.

Questo era il settembre a Meina. (Mondadori 1985: 53-56)

Quando una legge fascista impose la presenza del 20\% d'autori italiani in qualsiasi collana di letteratura, scegliendo chi dovesse confrontarsi con Wallace e Van Dine, nel 1931 Mondadori scelse Varaldo, che non poteva certo esser indifferente a una tipologia di romanzo che reinventava la tradizione dell'avventura e riformulava le tecniche dell'intrattenimento, un'opportunità per gli scrittori e una delizia per i lettori. Mondadori già nel novembre 1929 gli chiese qualche riga promozionale sulla letteratura poliziesca e sulla neonata collana «gialla», sicuro che lui, a differenza d'altri, non ne fosse «dispregiatore», ma anzi particolarmente predisposto come «i letterati inglesi che fanno le migliori feste a Wallace» ${ }^{9}$.

L'ammiratore però divenne presto autore e uscì Il sette bello, che nell'aprile del 1931 inaugurò la tradizione del «giallo» all'italiana, e che oggi sarebbe ancora godibile (qualcuno lo riprendesse in mano) ${ }^{10}$. La narrazione in prima persona, come da tradizione anglosassone, è qui riproposta cinque volte con i diversi punti di vista dei protagonisti: centrale (anche perché terzo) è quello del commissario Ascanio Bonichi, prima Giovanni Révere, studente per comodità, che apre il romanzo, e Maddalena Terzi, studentessa di medicina detta "Maud", poi altri due giovani come il bersagliere Biondo Biondi e il pittore Giacomo Serra.

\footnotetext{
${ }^{9}$ Lettera di Arnoldo Mondadori ad Alessandro Varaldo del 10 novembre 1929 (FAAM).

${ }^{10}$ Il romanzo nel giro di un decennio sparì dalla circolazione, vi tornò nel 1977 nei “GIM" Mondadori e nel 2006 con un'edizione a cura di Francesco De Nicola per De Ferrari.
} 
Ispirato probabilmente da un personaggio realmente esistito, un funzionario della stazione di Ventimiglia che col suo arrivo rese più sicuro quel caotico crocevia, Ascanio Bonichi, il primo detective italiano, baffuto e bonario, è decisamente differente dal Philo Vance di Van Dine, che è quasi un dandy, cultore dell'arte e studioso di psicologia, un Superuomo (Van Dine era un appassionato di Nietzsche), mentre il personaggio di Varaldo non ha doti particolari, è nei fatti un uomo medio. Decisamente differente anche dal Mr. Reader di Wallace, un investigatore trasandato abbastanza criminale per riuscire a interpretare e prevedere le mosse dei veri criminali, mentre Bonichi non ha la ben che minima dannazione e s'ispira piuttosto a un altro illustre detective, non nascondendo la propria «passione per Sherlock Holmes e i travestimenti classici di quel pioniere della deduzione» (Varaldo 1936a: 108).

Andrea Camilleri in qualche occasione ha confessato come senta "un vago odore" di Pasticciaccio davanti alle pagine dedicate a Bonichi, ma qui, forse per fortuna, non si ha lo spazio per districare una matassa tanto intricata e delicata anche se la mescolanza di codici linguistici (raffinato e romanesco, specialistico e popolare, francese e dialetto) darebbe qualche conforto in più al confronto fra due romanzieri così sproporzionatamente diversi come Varaldo e Gadda che solo scriverli sulla stessa riga fa tremare i polsi.

Si ritorni alla brasiliana Livraria do Globo che, dopo $O$ sete belo, pubblicò $O s$ sapatinhos vermelhos nel 1937, e un anno dopo A gata persa che uscì poi a metà degli anni Cinquanta anche per la portoghese Editorial Minerva, numero 33 della «Coleção Xis», e per l'argentina Editorial Kraft col titolo El caso de la gata persa e con la traduzione in spagnolo di José Garo, dopo aver già incluso nella stessa "Coleción Escorpión", con la traduzione di Emilio Lagorio, La desaparición de Rigel, quarto «giallo» di Varaldo, unico autore italiano presente nella collana (numeri 14 e 20) in cui Kraft pubblicò 24 romanzi polizieschi fra il 1954 e il 1957.

Non è banale l'operazione della Livraria do Globo, e nemmeno l'interesse dimostrato nei confronti del «giallista» italiano cui fu garantita una vetrina invidiabile, legittimata da alcuni nomi brasiliani d'indiscussa caratura. Os sapatinhos vermelhos, tradotto da Fulvia Bertolacci, per esempio, apparse sul popolare mensile $A$ novela diretto in quegli anni da Érico Verissimo, importante scrittore e fondamentale collaboratore della Livraria, con una splendida copertina disegnata da Edgar Koetz nella quale, benché nello stesso numero fosse incluso un romanzo del tedesco Karl May, campeggiano una scarpetta rossa e una collana di perle, nello sfondo un uomo con frac e revolver. $O$ sete belo e $A$ gata persa uscirono in volume e furono inseriti nella "Coleção Amarela» (37 e 59) e, se il primo fu tradotto da Luiz Estrella, il secondo da Mário Quintana, sensibile poeta, scrittore e traduttore della Woolf e di Proust e dei racconti di Sangue e parole di Giovanni Papini, inoltre coetaneo, connazionale e collega di Érico Verissimo col quale vivacizzò l'editrice Livrana do Globo e la stessa città di Porto Alegre.

Il poliziesco di Varaldo che ebbe più edizioni all'estero è quindi La gatta persiana. A fianco di Ascanio Bonichi sin dai tempi de Il sette bello, colta la propria prima occasione ne Le scarpette rosse, è il detective Gino Arrighi ad avviare senza volerlo, per volere del caso, le vicende del terzo «giallo»: sceso 
dall'autobus, passeggiando per una via di Roma, mentre sta andando a casa dalla moglie incinta (i suoi colleghi italiani e stranieri sono quasi tutti scapoli), vede un metronotte preoccupato per il miagolio triste di una gatta e va con lui a controllare da dove venga, per trovarsi poi davanti al corpo di un uomo che è il Sor Aronne, proprietario assassinato dell'animale e usuraio molto conosciuto nella capitale, il quale teneva un libro d'appunti che diverrà presto una lista dei sospettati.

L'influenza di Varaldo sul «giallo» all'italiana fu inevitabile, com'è evidente già nelle prime pagine de La gatta persiana: dallo stato civile di Gino Arrighi al suo arrivo coi mezzi pubblici, in un'ambientazione romana poco metropolitana, dall'importanza del caso all'impalcatura generale, passando dal cranio sfondato della vittima che è il massimo che l'autore conceda in termini d'immagini forti. E se il caso è sempre stato uno dei principali pilastri della narrativa varaldiana, qui nella produzione poliziesca sbroglia intrichi che mettono a dura prova l'autore (fra coincidenze improbabili e inevitabili incoerenze, oltre a sogni promontori, raptus sonnambuli ecc.) e soprattutto confondono il lettore che Varaldo stordisce per sorprendere. Loris Rambelli, che è stato uno dei primi ad analizzare il poliziesco varaldiano, poi oggetto di diversi studi di Francesco De Nicola, scrisse nel 1979 come «l'imprevedibilità e l'inverosomiglianza dello scioglimento finale» fosse «solo uno dei motivi di interesse dei gialli di Varaldo». (Rambelli 1979: 41)

L'italianizzazione del genere passò abbondantemente attraverso la produzione dello scrittore, così il «giallo» beneficiò della superficialità dell'analisi psicologica e della semplificazione dei ruoli, e s'impreziosì innocuamente di alcuni omaggi letterari che vanno dal dantesco «caddi come corpo morto» (Varaldo 1931a: 63) al manzoniano «se la mia spoglia fosse rimasta orba di tanto spiro» in Il segreto della statua, settimo poliziesco dell'autore (Varaldo 1936: 108), ma un poco rallentò nelle descrizioni e risentì delle digressioni. Inoltre Varaldo, non amando le scene troppo dure o gli scenari troppo crudi, finì per preferire i furfanti agli assassini. Effettivamente più che rispettare i moduli del poliziesco rilesse quelli personali, così già ne Le scarpette rosse poté, senza intaccare la riuscita del romanzo, fare a meno del delitto e condurre un'indagine sulla scomparsa di un paio di scarpette rosse, appunto, che contenevano i gioielli ed evocavano i ricordi di una ragazza una volta ricca, poi ridotta a dama di compagnia di una nobildonna napoletana (sia lecito citare Cenerentola in questo stesso saggio in cui si è citato già lo sceriffo di Nottingham). Infine si può aggiungere come i primi polizieschi varaldiani assomiglino a specie di feuilleton, come siano di un «giallo» più chiaro, quasi «rosa».

L'innesto «giallo»/Varaldo comunque funzionò, almeno coi germogli quando ai lettori appassionati della serie si sommarono i lettori fedeli all'autore. Mondadori poté ritenersi soddisfatto: Il sette bello raggiunse le 13 mila copie solo con la prima edizione, per poi arrivare, nel giro di un anno, a 23 mila con la seconda e la terza (poi 40 mila, una cifra importante per quei tempi). Tre ristampe (17 mila copie) ebbe anche Le scarpette rosse, confermando l'esordio di Varaldo giallista come un successo. Già nel 1933, sempre per Mondadori (a fine giugno l'autore confessava 
all'editore la propria scelta di non andare in vacanza per scrivere tutta l'estate) ${ }^{11}$ uscirono La gatta persiana e La scomparsa di Rigel, e questi primi quattro romanzi, come si è detto, sono quelli che furono tradotti in portoghese e spagnolo. Gli appassionati entusiasti in un primo momento dell'italianizzazione del genere tornarono a quegli stessi scrittori che lo resero grande, dal maestro Edgar Wallace, uno degli autori più letti del secolo, al redattore delle Twenty Rules for Writing Detective Stories, S. S. Van Dine, per citare i soliti.

Non bastò pubblicare la novità Casco d'oro (1936) al posto de Il tesoro dei Borboni, che era stato annunciato in chiusura di Circolo chiuso del 1935 e che, dopo una stesura travagliata, sarà pubblicato solo nel 1938. Comunque il destino del genere «giallo» era segnato poiché quello stesso regime fascista che ne obbligò l'italianizzazione presto lo vietò. E non ne poté nulla nemmeno Varaldo per quanto avesse perfettamente colto il nocciolo della questione, come si legge in alcune lettere a Mondadori: pur lamentandosi di certe limitazioni, rassicurava l'editore che i suoi «gialli» erano «moralissimi» e gli proponeva di pubblicare un volume più grosso (di racconti polizieschi) e soprattutto di venderlo a un costo maggiore, ponendo l'accento sul problema principale per il regime cioè la diffusione capillare e popolare di libri generalmente molto economici che potevano «dilagare» e raggiungere insomma, con i loro contenuti ritenuti scomodi, qualsiasi lettore (che si definirebbe anche elettore se negli anni Quaranta questa parola avesse avuto senso, meglio forse spettatore della grande messa in scena dell'Italia fascista senza macchia).

\section{LA «PROBABILITÀ» ED ESTAMPAS NAPOLEÓNICAS}

Seguendo la ricognizione sul riscontro spagnolo e portoghese dell'autore italiano si arriva al 1944, anno in cui furono pubblicati i due volumi di novelle De Ajaccio a Austerlitz e De Austerlitz a Santa Elena che compongono la raccolta Estampas napoleónicas, edita dalla casa editrice Morata di Madrid con la concessione di Garzanti e la traduzione di Pedro Fraga de Porto, il quale presentò una selezione del primo volume su Novelas y Cuentos ancora nel 1962.

$\mathrm{Fu}$ infinita e infaticabile la produzione novellistica di Varaldo: fra il 1919 e il 1946, in poco più di 25 anni, quasi 25 volumi, più di 250 racconti. Le avventure (Varaldo 1919b) e Una rosa d'autunno (Varaldo 1919c) uscirono nel 1919 tirando fra le 20 e le 40 mila copie e invitarono da subito a quella possibile, se pur generica, suddivisione delle novelle varaldiane fra vicende d'altri tempi e storie d'amore.

Mentre il genere sentimentale, rispettando sempre il buon gusto e il garbo, fondamentali per Varaldo, si risolve, nella brevità di poche pagine, in divertissement maliziosi e borghesi o, agli antipodi, in vicende di cuori palpitanti e occhi imperlati, il genere storico, partendo d'altri capisaldi dell'autore come la difesa della fantasia e la

11 Dalla lettera di Alessandro Varaldo ad Arnoldo Mondadori del 24 giugno 1933 (FAAM). 
lotta alla noia, si reinventa sfiorando l'aneddotica e si rafforza definendo la «probabilità», come all'universo varaldiano piacque definire, anche con il titolo della raccolta di novelle 23 probabilità del 1935 (Varaldo 1935a), la capacità del tempo passato di divertire il pubblico presente attraverso la caparbietà della Fantasia di riscrivere la Storia (nothing new under the sun direbbero gli inglesi).

Nell'intera prosa di Varaldo però, da un romanzo per ragazzi come Un fanciullo alla guerra (Varaldo 1917) ai volumi di racconti dedicati all'età napoleonica degli anni Trenta e Quaranta che saranno il culmine di questo genere, passando per la trilogia La marea (soprattutto ne Il falco) ma anche, pur con maggior rispetto della verità, in memorie e saggi come Maschere vive (Varaldo 1936b) e Pellegrinaggi letterari (Varaldo 1937), la «probabilità» ha intessuto vicende vere ad altre verosimili, ha indagato su personaggi politici come Carlo Alberto, Mazzini e Garibaldi, su pontefici e letterati italiani come Nievo, Leopardi e Prati, e stranieri, e li ha avvicinati a persone inventate (o meglio: intuite).

L'illuminazione poteva esser accesa da un dettaglio letto, da un aneddoto sentito $\mathrm{o}$, più spesso, dalla sua vorace lettura dei libri di storia. Da qui, dall'intuizione e dalla competenza, l'autore cominciava a costruire le sue «probabili» storie. Storie di mare e di teatro, cospirazioni e canzonature, le grandi figure e le loro ombre, le persone che stavano loro accanto o i piccoli fatti di poco conto: intesa in senso lato - politica, militare, letteraria e artistica in generale, ligure e marinaresca - la Storia si scheggia. E le scaglie si fan novelle, fantasiose e nel frattempo fedeli alla realtà, prodotti più riusciti della «probabilità», di cui sono quasi la palestra.

La figura certamente più amata è quella di Napoleone, che, dopo le comparsate ne La marea, diviene ben presto centrale. Le penne dell'aquila del 1928 sono esempio dell'interesse per l'imperatore e l'inclinazione alla "probabilità»:

L'impresa a cui mi volevo accingere mi piaceva, ma scritto il primo capitolo [...] m'accorsi che tutto avevo detto in poco spazio di carta; continuando avrei insistito sopra un tema unico, incappando nel pericolo monotonia [...]. Così, lasciato da canto il disegno primitivo, ecco il pullulare le novelle sui margini della storia napoleonica, e specialmente sui quei margini ov'era più facile riscontrar lacune o soluzioni di continuità, e quindi giocar di virtuosismo per riempirle. Volevo intitolare il mio racconto Aquila, come una specie di seguito al Falco; e invece mi sono dovuto accontentare delle penne, strappate o lasciate cadere, o perdute poco importa, ma penne, e non sempre delle ali. (Varaldo 1928: 11-14)

Passando per l'aggiornamento Nuove penne dell'aquila pubblicato da Mondadori (Varaldo 1935b) si arriva a Storie e leggende napoleoniche pubblicato da Garzanti nel 1942, che un paio di anni dopo, come si è detto, concederà i diritti a Morata per i due volumi, frutto di una forte rielaborazione almeno strutturale, di Estampas napoleónicas. Questi non a caso uscirono nella Colección Lyke che, piuttosto seriosamente, si proponeva di far luce sulle penombre della storia. Un'esagerazione che contagia anche il trafiletto introduttivo, in cui se si sbaglia la data di nascita dell'autore si dice almeno una cosa giusta sul volume quando si pone l'accento su come lo storico e l'aneddotico si fondano alla perfezione. 
Della "probabilità" Napoleone fu uno dei punti di partenza più sperimentati, e alcune fra le novelle napoleoniche sono i risultati più mirabolanti del genere "probabile"; questa l'«Avvertenza all'edizione» Garzanti, mantenuta in quella Morata:

Questa non è una biografia - tantomeno romanzata - di Napoleone Bonaparte.

Non è che una serie di scene, o di quadri, staccati in apparenza, uniti in sostanza da un unico filo conduttore. C'è la Storia, cioè dei fatti, che, più o meno riportarono contemporanei d'ogni tendenza, memorialisti d'ogni partito, e posteri che si vantarono d'essere imparziali. Questi fatti ho per lo più sottoposti ad una interpretazione, secondo la teoria della probabilità, e presentati pittoricamente, rispettando la prospettiva.

C'è la Leggenda, in massima creata dagli umili, dai soli disinteressati, dai soli in buona fede quindi [...]: la grande sacra falange dei minori fratelli ignoti.

Fra Storia e Leggenda mancava qualche volta un ponticello, ed ho cercato di gettarlo. Che il lettore vi passi sopra con fiducia, anche se lo pensi foggiato dalla fantasia. E veda soltanto la buona intenzione.(Varaldo 1942a)

In quegli stessi anni Varaldo su «Stampa Sera» tenne due rubriche chiamate Sul margine della storia (dal dicembre 1940 al gennaio 1942) e Avventure di tutti i tempi (dal febbraio 1942 al settembre 1943), quasi settanta novelle poi in parte confluite nei volumi Sul margine della storia (Varaldo 1944) e Leggende e storie dell'Ottocento (Varaldo 1947b) per Ceschina e Avventure e figure dell'Ottocento per Garzanti (Varaldo 1946a). Per Piccole storie di ieri e di oggi del 1946 (Varaldo 1946b) Varaldo come prefazione scrisse una lettera aperta all'amico e collega Tullio Giordana col quale molto stava discutendo della propria teoria della "probabilità", riepilogandola e confessando di sapere quanto potesse esser pericolosa come tecnica ma ormai era troppo tardi per rivedere un'intera carriera, chiosando con una citazione di Alfred de Musset, da sempre amatissimo dall'autore: «Mon verre n'est pas grand, mais je bois dans mon verre».

\section{CONFIDENZE E CONCLUSIONI}

Fra l'edizione milanese di Garzanti e quella madrilena di Morata dei racconti napoleonici, nel 1943, Andrés Guilmain, che dedicò quasi tutta la sua attività al genere umoristico, tradusse e inserì il breve racconto La dinastía de fantasmas di Varaldo nell'Antología de humoristas italianos contemporáneos pubblicata a Barcellona, che ospitava inoltre Pirandello, Moravia, Zavattini, Guareschi, Campanile, Palazzeschi, Trilussa, Pitigrilli, Bontempelli e Vergani. Nella nota biografica che precede la novella è ritardata la nascita di tre anni, ma in Spagna, si è detto, non è la prima volta che capita - e del resto anche in Italia a volte si trova 1878 al posto di 1876 -, e si elencano molte opere di Varaldo, ma stranamente non L'altalena, per poi concludere: «Literato de un gusto fino, depurado por una cultura sólida y enciclopédica, Varaldo es de la raza de narradores, indemne aún 
del microbio del cerebralismo a toda costa, y que escriben principalmente para interesar a sus lectores» (Guilmain 1943: 356). Si è sempre lì.

Inseribile nell'"umoristico" se non inteso stricto sensu, il racconto La dinastía de fantasmas è il rispolvero di parte del repertorio dell'autore nel ricorso al ricordo e nel ritmo del dialogo, nel castello isolato e misterioso e nelle caute concessioni al fantastico. Grazie a Guilmain nel 1956 la novella fu inserita nelle pagine centrali del numero di Novelas y Cuentos dedicato a Los buscadores de los tesoros di Irving Washington, e nel 1958 diede il titolo a un volumetto della Colección El Gorrión dell'Editorial G. P., occupandone solo le prime pagine. Il nome di Varaldo era l'unico sulla copertina ma nell'indice si leggono quelli di Pirandello (La tinaja e $L a$ señora Frola y el señor Ponza, su yerno), Zavattini (Un juego divertido e Un cuento de navidad), Campanile (Una aventura extraña, Llueve y Una noche infernal) e di altri in una selezione della già sommaria antologia del 1943.

Nello stesso 1943 su L'Illustrazione del Popolo apparse un lungo articolo dal titolo «Confidenze di Alessandro Varaldo». Qui l'autore ricordò il primo libro comprato con grande emozione e le prime ribellioni letterarie fra i banchi di scuola, ma soprattutto scrisse di «un bel signore con barba decorativa sul panciotto bianco», di «un generale, magro, asciutto e un po' nervoso» e di «una vecchia signora».

Racconta fu suo compagno di viaggio in treno, tratta Milano-Genova, il signore con la barba e il panciotto che leggeva. "Ci dormicchiava sopra, tra una stazione e l'altra, lo riprendeva e ricascava a sonnecchiare. Scese a Tortona dimentico del libro». Così lo scrittore decise che non avrebbe mai scritto pagine noiose. Il generale magro e nervoso è il secondo personaggio che, poeticamente e programmaticamente, incontrò sul treno, questo «leggeva, dando spesso segni di impazienza, un volume con la copertina grigia. Sbuffava e ricominciava qualche volta, come se non capisse, finché ad un tratto con un gesto risoluto scaraventò il libro dal finestrino». Così lo scrittore decise che non avrebbe mai scritto pagine irritanti. Stava partendo da Roma quando Varaldo, finalmente e felicemente, comprese che non aveva né annoiato né irritato i suoi lettori:

Nel vagone con me c'era una vecchia signora, che s'affacciava ogni poco al finestrino, con un'ansia evidente, come se attendesse qualcuno in ritardo. Mancavano pochi minuti alla partenza, quando giunse, ansando come una foca, un vecchio signore pingue [...] lieto in viso, agitando un volume giallo che riconobbi per il mio Sette Bello. Piombò nello scompartimento, e, senza occuparsi d'altro, gridò: - C'è davvero! E spiegò d'aver preso un tassi ed esser corso a Sant'Agnese per vedere il vicolo di Santa Costanza di cui parlo nel libro. (Varaldo 1943)

Non c'è dubbio che Varaldo sia riuscito nel suo intento. E il fatto che avesse appena pubblicato nel giro di pochi mesi (recano tutti la data 1942) ben quattro romanzi nuovi e due raccolte di novelle e per cinque editori diversi non è di poco conto. Oltre alla già citata Storie e leggende napoleoniche e L'avventura di Giuliana (Varaldo 1942b), i romanzi Il chiodo rosso per Mondadori (Varaldo 1942c), Senzaterra per Garzanti (Varaldo 1942d), L'ultimo dragone di Savoia per Ceschina (Varaldo 1942e) e Il soldato non è solo per Vallecchi (Varaldo 1942f). 50 
volumi uscirono di Varaldo solo fra le due guerre, e del resto già nel 1935 era uno degli autori più prolifici del suo tempo, se è vero com'è vero e come si legge in Scrittori nostri. Raccolta antologica di scritti inediti di Mondadori (VV. AA. 1935) che la sua scheda bibliografica aveva superato di gran lunga quelle degli altri autori inclusi, che erano 82, tra cui D'Annunzio, Pirandello e Marinetti. Oggi la bibliografia degli scritti di Varaldo che chi vi scrive, in quasi quattro anni di studi sull'autore, ha cercato di ricomporre nella sua totalità, fra poesia e teatro, romanzi e novelle, saggi e memorie, sfiora il centinaio di titoli e includendo la decina di prefazioni lo supera abbondantemente con le traduzioni dal francese e dei suoi libri in spagnolo e portoghese. Per non contare i contributi per la stampa periodica per i quali un centinaio di titoli non basta, se non nel suo doppio o triplo. Si recuperi la recensione di Possenti a Il covo:

Il Varaldo va accumulando opere su opere, con il fervore della formica di La Fontaine. Quando ci troveremo nella vallata di Giosafat nel famoso giorno sonoro di trombe apocalittiche $[\ldots]$ a ciascuno sarà chiesto conto, dalle Supreme Gerarchie del reparto letterario, del lavoro compiuto, scorgeremo Alessandro Varaldo farsi largo tra la folla degli scrittori e degli autori drammatici, tutti in costume da visita di leva, e presentarsi all'appello, $[\ldots]$ in gran daffare a sostenere due pile di opere. E così, con queste a stento contenute nell'ansa delle braccia, eretto il volto raso e alabastrino, smagato lo sguardo ceruleo, potrà proclamare con soddisfazione un numerosissimo elenco di pubblicazioni. Ma a noi pur beneauguranti, non è dato di penetrare gli imperscrutabili gusti delle supreme sfere... (Possenti 1927)

Varaldo riuscì a far abbattere alla propria carriera due barriere importanti e indicative come il 1900 e il 1950 con un ultimo studio dedicato al suo maestro Barrili, e riuscì appena a compiere il settantasettesimo anno di vita, essendo nato il 25 gennaio del 1876, che fu costretto a lasciar cadere l'altrimenti indomabile penna il 17 febbraio del 1953. Con le estreme ristampe italiane fu ancora una volta Mondadori a dimostrarsi la casa editrice più vicina a Varaldo (che comunque ebbe ottimi rapporti con molti editori che lo pubblicassero, come Ceschina e Treves, o meno, come Giovanni Scheiwiller), proponendo nella propria "Biblioteca Moderna" nel 1948 Il chiodo rosso del 1942, nel 1951 Un grand'uomo ed una piccola donna del 1935 (Varaldo 1935c), e infine nel 1952 I due nemici del lontano 1900 (cf. Varaldo 1900), il primo romanzo pubblicato in volume dall'autore, quasi a riprendere un discorso così iniziato e mai interrotto.

Il secondo conflitto mondiale recise in due il secolo ventesimo: i gusti della gente inevitabilmente cambiarono, quelli della critica inesorabilmente.

Tentare di prevedere i gusti invece delle «Supreme Gerarchie del reparto letterario» (per riprendere Possenti) forse è da stregoni, chissà avranno tempo durante il giudizio universale di leggere tutti i libri di Varaldo, se saranno gentili e pazienti da poter salvare il salvabile oppure tranchant e pigre da condannare l'autore senz'appello. Se non si tratta di un esercizio blasfemo, sicuramente è inutile, più opportuno è prendere atto di giudizi invece già espressi, come quelli dei lettori e dei critici, non all'altezza cronologica della fine del mondo ma di Varaldo, 
anzi prima dal momento, come si è detto, che le ombre dell'oblio che lo avvolse in uno sproporzionato silenzio dopo un così sintomatico successo, s'intravidero già in quel 1943 nel quale le Confidenze dell'autore ebbero un valore ormai riepilogativo e un sapore quasi epigrammatico: colui che non annoiò, colui che non irritò.

Troppo poco, almeno così è sembrato per molto tempo.

\section{RIFERIMENTI BIBLIOGRAFICI}

\section{Bibliografia degli scritti di Varaldo ai quali si fa riferimento}

GiRIBALDI, Alessandro; MALFetTANI, Mario; VARALdo, Alessandro (1897): $1^{\circ}$ Libro dei Trittici, Bordighera, Tipografia di Pietro Gibelli.

VARALDO, Alessandro (1898): Marine liguri, Milano, Aliprandi.

VARALDO, Alessandro (1900): I due nemici, Torino, Roux \& Viarengo [poi Milano, Sonzogno, 1919; "Libri azzurri", 12, Milano, Mondadori, 1931; "Biblioteca moderna", Milano, Mondadori 1952].

VARALDO, Alessandro (1904): Romanze e notturni, Milano, Libreria Editrice Nazionale.

VARALDO, Alessandro (1913a): Mio zio il diavolo, Milano, Baldini \& Castoldi [poi "Libri azzurri", 68, Milano, Mondadori, 1923 e 1932,].

VARALDO, Alessandro (1917): Un fanciullo alla guerra (Avventura di un fanciullo nella campagna del 1848), Milano, Treves [poi "Il libro dei ragazzi", 12, Milano / Imperia, 1923; Milano, Ceschina, 1929].

VARALDO, Alessandro (1913b): Genova sentimentale, Genova, Libreria Editrice Moderna.

VARALDO, Alessandro (1919a): La bella e la bestia, Milano, Sonzogno [poi Milano, Ceschina, 1930].

VARALDO, Alessandro (1919b): Le avventure, "Le Spighe", 13, Milano, Treves,.

VARALDO, Alessandro (1919c): Una rosa d'autunno, Milano, Treves [poi Milano, Sonzogno, 1920].

VARALDO, Alessandro (1920a): La grande passione, Milano, Sonzogno [poi "Libri azzurri", 85, Milano, Mondadori, c.1923 e 1933,].

VARALDO, Alessandro (1920b): L'ultimo peccato, Milano, Vitagliano [poi Milano, Sonzogno, 1922; "Libri azzurri", 101, Milano, Mondadori, 1933].

VARALDO, Alessandro (1920c): La costa azzurra, Milano, Sonzogno.

VARALDO, Alessandro (1921): I cuori solitari, Milano, Vitagliano [rielaborazione de I Signori di Nervia (64 puntate apparse sul genovese Supplemento del Caffaro dal 6 luglio al 9 settembre 1898); poi Milano/Roma, Mondadori, 1924].

VARALDO, Alessandro (1922): Il falco (Cronaca del 1796), Milano/Roma, Mondadori.

VARALDO, Alessandro (1925a): L'amante di ieri, Milano, Mondadori [poi "Libri azzurri", 142, Milano, Mondadori 1926].

VARALDO, Alessandro (1925b): Il fior d'agave, Milano, Sonzogno.

VARALDO, Alessandro (1927a): Il covo, Milano, Mondadori. 
VARALDO, Alessandro (1927b): «Quel che dice e quel che fa... Alessandro Varaldo», Almanacco letterario, Milano, Mondadori, pp. 288-289.

VARALDO, Alessandro (1928): Le penne dell'aquila (Cronache dell'epopea napoleonica), "Libri azzurri", 72, Milano, Mondadori.

VARALDO, Alessandro (1931a): Il sette bello, "Libri gialli", 21, Milano, Mondadori, [poi "Gialli Italiani Mondadori”, 1, 1977; Genova, De Ferreri, 2006]

VARALDO, Alessandro (1931b): «Caduta e resurrezione di Figaro», Comoedia, XIII/9, pp. 9-10

VARALDO, Alessandro (1931c): Le scarpette rosse, "Libri gialli", 28, Milano, Mondadori. [poi "Gialli Italiani Mondadori", 5, Milano, Mondadori, 1977].

VARALDO, Alessandro (1933b): La gatta persiana, "Libri gialli", 58, Milano, Mondadori. [poi "Gialli Italiani Mondadori", 9, Milano, Mondadori, 1977].

VARALDO, Alessandro (1933c): La scomparsa di Rigel, "Libri gialli", 139, Milano, Mondadori.

VARALDO, Alessandro (1935a): 23 probabilità, "PandoraVoci di tutti i popoli", 8, Milano, Sperling \& Kupfer,

VARALDO, Alessandro (1935b): Nuove penne dell'aquila, "Libri azzurri", 146, Milano, Mondadori,.

VARALDO, Alessandro (1935c): Un grand'uomo ed una piccola donna, Milano, Mondadori [ristampato in edizione integrale, "Biblioteca Moderna Mondadori" 11-bis, 1951].

VARALDO, Alessandro (1936a): Il segreto della statua, Milano, "Libri gialli”, 139, Mondadori,.

VARALDO, Alessandro (1936b): Maschere vive. Storie e cronache di palcoscenico, Milano, Ceschina.

VARALDO, Alessandro (1937): Pellegrinaggi letterari, Milano, Mondadori.

VARALDO, Alessandro (1938): Il tesoro dei Borboni, "Libri gialli", 194, Milano, Mondadori.

VARALDO, Alessandro (1939): L'altalena, Milano, Ceschina.

VARALDO, Alessandro (1942a): Storie e leggende napoleoniche, Milano, Garzanti.

VARALDO, Alessandro (1942b): L'avventura di Giuliana, Roma, Edizioni Stella.

VARALDO, Alessandro (1942c): Il chiodo rosso, Milano, Mondadori [poi "Biblioteca Moderna Mondadori", 18, 1948,].

VARALDO, Alessandro (1942e): Senza terra, Milano, Garzanti.

VARALDO, Alessandro (1942f): L'ultimo dragone di Savoia, Milano, Ceschina.

VARALDO, Alessandro (1942g): Il soldato non è solo, Firenze, Vallecchi.

VARALDO, Alessandro (1943): «Confidenze di Alessandro Varaldo», L'Illustrazione del Popolo XXIII/23, 6 giugno, p. 9.

VARALDO, Alessandro (1944): Sul margine della storia, Milano, Ceschina.

VARALDO, Alessandro (1946a): Avventure e figure dell'Ottocento, Milano, Garzanti.

VARALDO, Alessandro (1946b): Piccole storie di ieri e di oggi, Milano, Cavallotti. VARALDO, Alessandro (1947a): «Tragico e buffo figuro Mussolini visto da Senise», Giornale d'Emilia, III/32, 2 febbraio, pp. 1-2. 
VARALDO, Alessandro (1947b): Leggende e storie dell'Ottocento, Milano, Ceschina.

\section{Scritti di Alessandro Varaldo tradotti in spagnolo e portoghese}

VARALDO, Alessandro (1921): «Ardillas y lirones», trad. sp. di José Lopez Pinillos e Enrique Tedeschi, Los contemporáneos, año XIII, núm. 634.

VARALDO, Alessandro (1931): El gato negro, trad. sp. di Tirso Lorenzo, Buenos Aires, Las comedias de El Hogar, giugno.

VARALDO, Alessandro (1935): O sete belo, tr. port. di Luiz Estrella, "Coleção Amarela", 37, Porto Alegre, Livraria do Globo,.

VARALDO, Alessandro (1937): Os sapatinhos vermelhos, tr. port., "A novela" 14, di Fulvia Bertolacci, Porto Alegre, Livraria do Globo.

VARALDO, Alessandro (1938): A gata persa, tr. port. di Mário Quintana, Coleção Amarela", 59, Porto Alegre, Livraria do Globo, ".

VARALDO, Alessandro (1943): La dinastía de fantasmas, tr. sp. di Andrés Guilmain, in A. Guilmain / G. B. Ricci, J. Janés (eds.), Antología de humoristas italianos contemporáneos, Barcelona, N.A.G.S.A, pp. 365-376.

VARALDO, Alessandro (1944): Estampas napoleónicas I (De Ajaccio a Austerlitz), tr. sp. di Pedro Fraga de Porto, Madrid, Morata. Primera parte. La pequeña sombra include La corona de Hierro (1755); Y, árbitro, se sienta (1774-1814); Los ojos azules (1774-1812); La lacción memorable (1779); La primera lección (1779-1814); La lección de historia (1780-1800); El «tio» de Versailles (17801812); Bajo el rajo lunar (1791-1815); Un duelo (1791); La deuda pagada (1791-1804); Dos palabras (1792); La cura de la insolación (1792-1806); La Fortuna (1892-1815); Dos viejos amigos (1792-1804); Basta! (1792-1820); El bloqueo (1792-1820). Segunda parte. El general jefe include La diligencia verde (1796-1802); La estrella (1896); Historia de una tabaquera (1796-1832); La maestra Codogni (1796); Los dos vados (1796-1798); Sinfonía heroica (1796); Una frase famosa (1796); Diversidad (1796-1859); La nariz de Cleopatra (1896-1814); El hombre honrado (1796); Las tres rosas de la emperatriz (17961814); La transfiguración (1796-1816); Las pistolas del general (1797-1860); El último puente de Oudinot (1798-1840). Tercera parte. El primer cónsul include Zerlina (1899-1814); El Orden (1799); La espada número 147 (1792-1802); El padre de don Beltrán (1800-1815); La bendición (1800-1848); El bandolero (1800); El tesoro (1800-1814); Dos prisoneros (1800-1815); Los tres amores de la abuela (1795-1851); Una botella de aguardiente (1800); La buena mentira (1801-1805); El pedestal (1804).

VARALDO, Alessandro (1944): Estampas napoleónicas II (De Austerlitz a Santa Elena), trad. sp. di Pedro Fraga de Porto, Madrid, Morata. Cuarta parte. El emperador include La víspera de Austerlitz (1805); La camana de Navidad (1805); El rodevo prudente (1806); Como una mujer se empeñe (1806); La ballena (1806-1811); El hombre y el soledad (1806); El italiano (1807); La fidelidad (1807-1837); La gratitud (1808-1814); La última colección (1808- 
1840); La fuerza ignota (1808); La dentelladas (1809); Bernardo-Joaquín (1809-1821); Padre y hijo (1809-1821); Los asuntos serios (1809-1815); Una profecía (1809-1814); Carácter de soldado (1809); Un vaso de agua azucarada (1811-1813); El pastel de cerezas (1811-1822); El rosario (1812); Los melocotones en Navidad (1814-1815). Quinta parte. La gran sombra include La gran sombra (1814-1848); Los dos carceleros (1821); El arlequín (1821); Una bandera (1821); Un trocito de cinta (1821); El Emperador retorna (1815-1830); El cielo azul (1831); El regreso del marido (1817-1832); Una noche en el Coliseo (1839).

VARALDO, Alessandro (1954): A gata persa, trad. port. di Gaspar Natividade, Lisbona, "Coleção Xis", 33, Minerva.

VARALDO, Alessandro (1955): La desaparición de Rigel, trad. sp. di José Garo, "Colleción Escorpion", 14, Buenos Aires, Guillermo Kraft.

VARALDO, Alessandro (1956 ca.): El caso de la gata persa, trad. sp. di Emilio Lagorio, "Colección Escorpion", 20, Buenos Aires, Gullermo Kraft,.

VARALDO, Alessandro (1956): «La dinastía de fantasmas», trad. sp. di Andrés Guilmain, Madrid, Novelas y Cuentos, XXVIII/1329, pp. 17-18 [le pagine centrali; il fascicolo contiene Los buscadores de tesoros di Washington Irving].

VARALDO, Alessandro (1958): La dinastía de fantasmas, tr. sp. Andrés Guilmain, in La dinastía de los fantasmas, "Colección El Gorrión", 14, Barcelona, Editorial G. P.

VARALDO, Alessandro (1962): «Estampas naoleónicas (De Ajaccio a Austerlitz)», trad. sp. di Pedro Fraga de Porto, Madrid, in Novelas y Cuentos, XXXIV/1639 [selezione di novelle dal volume I Morata,1944].

\section{Studi su Varaldo e altri riferimenti bibliografici}

ALCÁZAR, Luis (1924): «¿Sociedad de autores o de recaudadores?», La Libertad, $\mathrm{VI} / 1433,2$ novembre, $\mathrm{p} .4$.

ALSINA, José (1920): «Ardillas y lirones», in El Sol, IV/800, 26 febbraio, p. 9.

CHABÁs, Juan (1926): «Recuerdos literarios», La Libertad, VIII/2073, 3 settembre,p.5.

CHABÁS, Juan (1927a): «Almanacco literario 1927 Mondadori», La Gaceta Literaria, $\mathrm{I} / 3,1^{\circ}$ febbraio, p. 4.

CHABÁs, Juan (1927b): «Il covo», La Libertad, IX/2156, 18 febbraio, pp. 6-7.

CHABÁs, Juan (1928): Italia fascista, Barcelona, Mentora.

DE ANGELIS, Augusto (1925): "Ritratti in lapis: "I due Varaldo"», Comoedia, VII/14, 15 luglio, pp. 727-730.

DE NiCOLA, Francesco (1992): Dal best seller all'oblio, Genova, Marietti.

Diderot, Denis (1909): Paradosso sull'attor comico, tr. it. e pref. di Alessandro Varaldo, Milano, Sonzogno.

DOUGHERTY, Dru; VICHES, Maria Francisca (1990): La escena madrileña entre 1918 y 1926. Análisis y documentación, Madrid, Editorial Fundamentos.

F[?]. (1920): «Estreno de Ardillas y lirones», $A B C, \mathrm{XVI} / 5346,26$ febbraio, p. 15. 
FERNÁNDEZ AlMAgro, Melchor (1920): «Ardillas y lirones», Época, LXXII/24.909, p. 1.

FERRARO, Alessandro (2011): "Alessandro Varaldo l'autore ritrovato», in Alessandro Varaldo, La marea, Massa, Transeuropa, pp. ix-xxxiii.

GRAMSCI, Antonio (1953): Letteratura e vita nazionale, Torino, Einaudi.

GRUPPO DEI DIECI [Massimo Bomtempelli, Lucio D'Ambra, Alessandro De Stefani, Filippo Tommaso Marinetti, Fausto Maria Martini, Massimo Milanesi, Alessandro Varaldo, Cesare Giulio Viola, Luciano Zuccoli] (1928 [2011a]): Il Novissimo segretario galante. Approcci, Roma, Sapientia Edizioni dei Dieci. [Nuova ed. in Roma, Graphofeel - lettere selezionate].

GRUPPO DEI DIECI [Massimo Bomtempelli, Lucio D'Ambra, Alessandro De Stefani, Filippo Tommaso Marinetti, Fausto Maria Martini, Massimo Milanesi, Alessandro Varaldo, Cesare Giulio Viola, Luciano Zuccoli] (1929 [2005]): Lo zar non è morto, Roma, Edizioni dei Dieci. [Nuova ed. in Milano, Sironi].

LuCIGNANI, Luciano (1984): «La resa di D’Annunzio», Stampa Sera, CXVIII/152, 28 giugno, p. 9.

MACHADO, Manuel (1920): «Ardillas y lirones», La Libertad, II/71, 26 febbraio, p. 3.

MARINI, Leonardo (1921): «Crónica de Italia. El teatro, los libros y le arte», Cosmópolis, II, pp. 319-324.

MONDADORI, Mimma (1985): Una tipografia in paradiso, Milano, Mondadori.

MORI, Arturo (1920): «Ardillas y lirones», El País, XXXIV/11.819, 26 febbrario, p. 1.

PARODI, Mario (1953): «Ultima sosta di Varaldo a Genova», Genova, XXXIII/2, febbraio, p. 34-38.

PÉREZ BAZO, Javier (1992): Juan Chabás y su tiempo: de la poética de vanguardia a la estética de del compromiso, Barcelona, Anthropos.

PICENI, Enrico (1928): La bancarella delle novità, Milano, Alpes.

PoSSENTI, Eligio (1927): «Varaldo e il "Covo"», Corriere della Sera, 14 luglio, p. 3

PRAGA, Marco (1921): «Un marito innamorato», Cronache teatrali: 1920, Milano, Treves, pp. 248-254.

RAMBELLI, Loris (1979): «Alessandro Varaldo o dei vecchi merletti», Storia del "giallo» italiano, Milano, Garzanti, pp. 31-46.

RODRÍGUEZ PUÉRTOLAS, Luis (1986): Literatura fascista española, Madrid, Akal, vol. I.

SÁNCHEZ MAZAS, Rafael (1928a): «Novísimo secretario galante», $A B C$, XXIV/ 7952, 23 giugno, pp. 6-7.

SÁNCHEZ MAZAS, Rafael (1928b): «Una reunión de "los diez"》, $A B C$, XXIV/ 7978, 24 luglio, pp. 7-8.

SIMONI, Renato (1931): «Alessandro Varaldo. Un fecondo creatore di intrecci romanzeschi», Stampa Sera, LXV/42, 18 settembre, p. 3.

SQUARZINA, Luigi (1987): Ciascuno a suo modo di Pirandello e il teatro totale delle avanguardie, Roma, Bulzoni. 
TEDESCHI, Enrique (1922): «Por estrenar un drama», El Imparcial, LVI/19.688, 10 marzo, p. 3.

VV. AA. (1935) Scrittori nostri. Raccolta antologica di scritti inediti, Milano, Mondadori. 